\title{
Gender and ethnic disparities contributing to overweight in California adolescents
}

\author{
Mary Ellen Wilkosz • Jyu-Lin Chen • \\ Christine Kennedy $\cdot$ Sally Rankin
}

Received: 14 May 2009 / Accepted: 24 September 2009 /Published online: 1 December 2009

(C) The Author(s) 2009. This article is published with open access at Springerlink.com

\begin{abstract}
Purpose To explore differences in health behaviors and factors contributing to overweight among 12 to 17 year olds in California.

Methods Data from the 2005 California Health Interview Survey for 3,315 adolescents self-identified as Latino, Asian, or white were reviewed. Adolescents reported their weight, height, gender, ethnicity, parents' educational level, household income, physical activity, sedentary activity, breakfast consumption, and family meals.

Results Overall $34 \%$ of boys and $22 \%$ of girls in this study were overweight ( $>85$ th percentile for age and gender). Approximately $38 \%$ of Latinos, $25 \%$ of whites, and $16 \%$ of Asians were overweight. Latinos were more than twice as likely to be overweight as whites (2.07) and Asians (2.53). Younger adolescents (12-13 years old) and adolescents whose family income is less than $200 \%$ of the federal poverty level were more likely to be overweight. Low level of parental education is a risk factor for Latino and Asian girls and white and Latino boys. White girls with a lower socioeconomic
\end{abstract}

Funding Support from the UCLA Center for Health Policy Research, UCSF Century Club, Sigma Theta Tau International Chapter Lambda Gamma.

This publication was made possible by grant no. UL RR024131-01 from the National Center for Research Resources (NCRR), a component of the National Institutes of Health (NIH) and NIH Roadmap for Medical Research.

M. E. Wilkosz $(\bowtie)$

Department of Nursing, Sonoma State University, 1801 E. Cotati Ave., Rohnert Park, CA 94928, USA

e-mail: wilkosz@sonoma.edu

J.-L. Chen · C. Kennedy $\cdot$ S. Rankin

Department of Family Health Care Nursing,

University of California,

San Francisco, CA 94143, USA status and white boys with more than $2 \mathrm{~h}$ daily of television, video, and computer time were more likely to be overweight. Conclusion Results suggest gender and ethnic variations in factors that contribute to overweight in California adolescents. To influence the current overweight epidemic, clinicians must develop culturally sensitive and genderspecific interventions that address the unique needs of an ethnically diverse adolescent population.

Keywords Gender - Ethnicity · Adolescent · Overweight . Risk factors

\section{Introduction}

The prevalence of obesity remains a health concern for children and adolescents in the United States. During the combined years of 2003 to 2006, an estimated $16.3 \%$ of children and adolescents from 2 to 19 years old were overweight, at or above the 95th percentile body mass index (BMI) according to age growth charts from the National Health and Nutrition Examination Survey determined in 2000 (CDC 2009). Nationally, 17.1\% of children and adolescents are overweight (Ogden et al. 2006), and these rates are even higher in California (18.9\%) (AskCHIS 2009). Health consequences of overweight in adolescents, including early heart disease, diabetes, and psychological ramifications such as teasing, discrimination, and victimization, make prevention a priority (Atabek et al. 2006; Must et al. 2007; Rowland 1999).

Nationally, Asian Americans as a whole are at lower risk for overweight $(20.6 \%)$ than are African Americans $(30.9 \%)$, Latinos $(30.4 \%)$, and whites $(24.2 \%)$. A similar trend is also found in California (Asian Americans, 11.3\%; African Americans, 21.5\%; Latinos, 24.4\%; whites, 13.9\%) (AskCHIS). However, according to the National Longitu- 
dinal Study of Adolescent Health, with an increase from $11.6 \%$ to $28 \%$, Asian American children born in the second and third generation have the largest and most significant increase in the incidence of overweight compared with children born in the first generation (Popkin and Udry 1998). Similar trends were found in Hispanic American adolescents, with an increase from $24.5 \%$ to $31.7 \%$ from the first generation to the second and third generation (Popkin and Udry). Thus, disparities exist among overweight prevalence, especially among minority adolescents, yet the causes of these disparities are unclear (Ogden et al. 2008).

Several theories have been used and proposed to examine childhood obesity, including the ecological systems theory. The basic premise of the ecological systems theory is that individuals and their environment have a dynamic interaction and relational nature (Laustsen 2006; Davison and Birch 2001). Ecological models of health behavior focus on individual influences such as physical activity and sedentary activity, as well as on social (family meals) and environmental factors (access to parks and bike and hiking trails) that may facilitate or inhibit individual behavior (Sallis and Owen 1997). Possible causes of ethnic differences in weight include socioeconomic status (Drewnowski and Damon 2005), gender, education level of parents, consumption of breakfast, family meals (Neumark-Sztainer et al. 2003; Woodruff and Hanning 2009), amount of sedentary activity, and amount of physical activity (Nader et al. 2008). The literature supports the multifactoral nature of obesity, and the ecological model supports the multiple levels of influence that determine individual behavior. In this study, the ecological model was used to examine factors (including variables mentioned in the model) related to overweight in adolescents in California.

Latinos and Asian Americans are rapidly growing populations of immigrants in California and in the US and are understudied populations, making it critical to examine specific factors in these populations. A better understanding of overweight-related health behaviors and sociocultural differences among Latino, Asian American, and white adolescents in California will help in developing culturally sensitive interventions directed at the overweight epidemic. Thus, the purpose of this study was to use the 2005 CHIS data to explore differences in overweight-related health behaviors between genders and among ethnicities in an effort to understand better the factors that contribute to overweight in 12 to 17 year olds in California.

\section{Methods}

\section{Overview}

The CHIS 2005 is a large population-based, cross-sectional, biennial health survey in California that is the largest state survey in the US. The California Health Interview Survey (CHIS) 2005 was implemented to determine the prevalence of and trends in health-related behaviors among adults $(\mathrm{n}=$ $43,020)$, adolescents $(n=4,029)$, and children $(n=11,358)$ in California (CHIS 2007; Lopez-Zetina et al. 2006). The present analysis reports findings related to the population of 12 to 17 year olds in California. The CHIS is the largest health survey in the nation and is a model for other states that are developing population-based tools for monitoring public health (Holtby et al. 2008). The CHIS is recognized as an essential source of ongoing public health data for the state, counties, and different ethnic groups in California's noninstitutionalized population living in households. The survey and data collection are conducted by the University of California, Los Angeles, Center for Health Policy Research, in conjunction with the California Department of Health Services and the Public Health Institute (Brown et al. 2005, p.333). A detailed description of the CHIS methodology has been published elsewhere (CHIS 2007a).

\section{Sampling design}

The objective of the CHIS sampling design is to provide health-related estimates for the overall population in California, the largest ethnic/racial groups, and several smaller ethnic/racial groups of interest. Information collected in the CHIS 2005 included the occurrence of healthy and unhealthy behaviors, exposure to potential risk factors, dietary intake, physiological measures (height, weight), health care services and utilization, the incidence and prevalence of disease, and other health-related factors. Data are input directly into a computer during the interview process. The sample purposefully represents the ethnic diversity of California to increase generalizability. The data were collected for this survey between July 2005 and April 2006.

The sample design for CHIS 2005 was complex, requiring weighting to make accurate population estimates. The CHIS sample was designed to yield estimates for most counties in California and for the state's major ethnic and racial groups as well as for numerous smaller racial and ethnic populations. Certain ethnic groups (Korean and Vietnamese) were purposefully over sampled by using telephone numbers associated with group-specific surnames drawn from listed telephone directories to increase the sample size further (CHIS 2007b). The survey's large sample size and administration in five languages, including English, Spanish, Chinese (Mandarin and Cantonese dialects), Korean, and Vietnamese, helps to capture the diversity of the California population. These languages were chosen on the basis of analysis of data from the 2000 census to identify the languages that would cover the largest number of Californians, thus improving the chances of the CHIS representing California's diverse population 
(CHIS 2007a; Ponce et al. 2004). Approximately 10\% of the adult interviews, $18 \%$ of the child (parent proxy) interviews, and $7 \%$ of all adolescent interviews were completed in a language other than English (CHIS 2007b).

\section{Data collection}

Survey data were collected by using a multistage random digit-dial telephone survey, with 41 geographically defined sampling strata, a large sample, and purposeful over sampling of several ethnic populations. In those households with adolescents ( $12-17$ years old) and/or children $(<12$ years old), one adolescent and one child were randomly selected; the adolescents were interviewed directly after consent for participation was obtained from the parent, and the adult most knowledgeable about the child's health completed the child interview (CHIS 2007a). Data were collected on 43,020 adults and 4,029 adolescents. Detailed descriptions of the sampling and data collection methods can be found in the CHIS 2005 Methodology Series, posted on the web at http://www.chis.ucla.edu.

\section{Response rates}

The overall response rate for CHIS 2005 was created by combining the screener completion rate (successful introduction of survey to a household and randomly selecting an adult to be interviewed) and the extended interview completion rate (successfully getting one or more selected persons to complete the extended interview) (CHIS 2007a). For CHIS 2005, letters explaining the survey in five languages were sent out in advance to $67 \%$ of the sampled telephone numbers to maximize response rates (CHIS 2007a). The household extended interview response rate was $59.3 \%$, which is comparable to other random-digit-dial surveys. Overall extended interview response rates for were $48.5 \%$ for adolescents and $75.2 \%$ for children (CHIS 2007).

\section{Study variables}

\section{Demographic}

The purpose of the study was to explore the ethnic and gender differences in factors contributing to overweight in adolescents participating in the CHIS. Based on the ecological model, questions related to gender, age, parents' educational level, household income, physical activity, sedentary activity, breakfast consumption, and family meals were selected for analysis.

Twelve- to 17-year-old adolescents self-reported their age, gender, and race/ethnicity. The variable for age was categorized to include groupings as follows: 12 to 13 years old, 14 to 15 years old, and 16 to 17 years old. Inclusion in this analysis was based on self-report of specific ethnicities including Latino, Asian American, and white; all other selfreported ethnic groups were excluded from analysis (inclusion of Asian and Latino adolescents was based on the fact that they are the two fastest growing minorities in California and have been understudied in the current literature). Socioeconomic status was explored by using the percentage of federal poverty level (FPL) with ranges of $0 \%$ FPL to $99 \%$ FPL (lowest income group), 100\% FPL to $199 \% \mathrm{FPL}, 200 \% \mathrm{FPL}$ to $299 \% \mathrm{FPL}$, and greater than $300 \%$ FPL (highest income group).

\section{Parent educational level}

Parent educational level (education level of parent interviewed) was based on the question "What is the highest grade of education you have completed and received credit for?" This information was reported by parents before or immediately after the adolescent was interviewed. This variable originally had 11 categories (no formal education, grade 1-8, grade 9-11, grade 12/high school diploma, some college, vocational school, associate's degree, bachelor's degree, some graduate school, master's degree, doctorate or equivalent). Parent educational level was recoded into four categories: less than high school, high school graduate, some college, and college degree or higher.

\section{Outcome/BMI and overweight status}

Adolescents self-reported their height and weight, and then BMI was calculated (BMI = weight in kilograms divided by height in meters squared). This variable was created by taking the self-reported height and weight for each participant and then using the BMI to determine age- and gender-specific percentiles for the BMI. BMI between the 5 th and the 84th percentile were considered to be "normal weight," and BMIs at the 85th percentile and higher were considered "overweight" according to the CDC's criteria from 2000 (CDC 2009a).

\section{Physical activity}

Adolescents were asked questions related to physical activity including "Over a typical week, on how many days are you physically active for at least $60 \mathrm{~min}$ total per day?" The physical activity variable was recoded depending on whether the adolescent met the American Pediatric Association's (APA's) guidelines for physical activity, which is a minimum of $60 \mathrm{~min}$ of moderate physical activity for at least 3 days per week. The continuous variable was recoded to a dichotomous variable yes ( $\geq 3$ days of physical activity for $60 \mathrm{~min} /$ day) or no ( $<3$ days of physical activity for $60 \mathrm{~min} /$ day). 


\section{Sedentary activity}

For sedentary activity, adolescents were asked to "think about your free time on Monday through Friday, on a typical day, about how many hours do you usually watch TV or play video games?" and "about how many hours per day on Monday through Friday do you use a computer for fun, not schoolwork?" These same questions related to sedentary activity were asked about a typical Saturday and Sunday. First television/video time and computer time were added together for the Monday through Friday time period, and the same was calculated for the Saturday through Sunday period. The average daily number of hours spent on these sedentary activities was calculated as a weighted average of weekday and weekend responses. A new variable called screen time was created that summarized sedentary activity in each adolescent, dichotomizing this number into meeting the APA's guideline for sedentary activity or not meeting the APA's guidelines (APA recommends no more than $2 \mathrm{~h}$ of screen time per day). The screen time variable allowed easy comparison with the physical activity guidelines.

\section{Breakfast consumption}

Another question posed that related to health-promoting behaviors involved breakfast consumption. Adolescents were asked, "In the past 7 days, how many days did you eat breakfast?" The number of days that breakfast was eaten was dichotomized to create a yes or no response to whether breakfast was eaten in the morning. Responses of 0 to 6 days were dichotomized as no and 7 days was dichotomized as yes.

\section{Family meals}

Adolescents were asked, "In the past 7 days, how many days did you eat dinner at home with at least one of your parents (guardians)?" The variable related to eating dinner at home with at least one parent or guardian was also dichotomized as no for 0 to 6 days and yes for 7 days.

\section{Statistical analysis}

In order to represent the study population, 80 replicate weights and a final weight variable were used to generate descriptive statistics for all of the study variables, means and standard deviations for the continuous variables, and frequency and percentages for categorical variables.

Univariate logistic regressions were used for each of the independent variables (gender, ethnicity, physical activity, sedentary activity, breakfast consumption, family meals, age, parents' educational level, and household income) to predict the dichotomous overweight outcome variable (overweight vs not overweight). The relationships between these independent variables and overweight status were also examined through the alternative strategy of contingency tables and the chi-square statistic. A multivariate logistic regression was then performed to examine the interrelationships among all of the independent variables simultaneously and overweight status. Potential interactions between gender or ethnicity and the other independent variables were also explored in the multiple logistic regression analysis. All analyses were performed by using SPSS/PC statistical program (version 10 for Windows; STATA, College Station, Texas). A $p$ value of 0.05 or less was accepted as significant.

\section{Results}

General characteristics

A total of 3,315 California adolescents from 12 to 17 years old were included in the analysis. Tables 1 and 2 present information about the characteristics of white (51.4\%), Latino (35.0\%), and Asian American (13.6\%) adolescents participating in the 2005 CHIS. Overall, parents of adolescents in the 2005 CHIS study had high levels of education attainment $(56.7 \%$ with some college or a college degree and higher). Only 16.2\% reported incomes at or below the poverty level, and almost half reported incomes at or above $300 \%$ of the poverty level.

Less than $20 \%$ of the respondents met the APA's recommendation of $2 \mathrm{~h}$ or less of screen time per day (combination of TV, computer, and video games). Asian American adolescents had the highest percentage of respondents exceeding $2 \mathrm{~h}(87 \%$ for girls and 91\% for boys). Only $26 \%$ of respondents did not meet the APA's recommendations for physical activity (60 min of moderate physical activity $>3$ days/week). Again, Asian American adolescents had the highest percentage of respondents not meeting the APA's recommendation for physical activity (43\% for girls and 37\% for boys). The mean number of days that respondents were physically active for a minimum of 60 min per day was 3.90 (SE, 0.05). Mean screen time for respondents was 3.82 hours per day (SE, 0.07). More than 50\% of respondents reported not eating breakfast on a daily basis, particularly white girls and Latinas. Forty-five percent of respondents reported not eating dinner with a parent or guardian daily, and this pattern was seen more often in white and Latino girls and boys. Table 3 summarizes the prevalence of the independent variables described here on the basis of gender and ethnicity. 
Table 1 Percentage distribution by selected characteristics: California adolescents, California health interview survey, 2005

\begin{tabular}{|c|c|c|}
\hline & $\begin{array}{l}\text { Number }^{\mathrm{a}} \\
(\mathrm{N}=3,315)\end{array}$ & $\%{ }^{\mathrm{a}}$ \\
\hline \multicolumn{3}{|l|}{ Race/ethnicity } \\
\hline White & 2,135 & 51.4 \\
\hline Latino & 827 & 35.0 \\
\hline Asian American & 353 & 13.6 \\
\hline \multicolumn{3}{|l|}{ Age, years } \\
\hline $12-13$ & 1,113 & 32 \\
\hline $14-15$ & 1,172 & 35.3 \\
\hline $16-17$ & 1,030 & 32.7 \\
\hline \multicolumn{3}{|l|}{ Gender } \\
\hline Males & 1,694 & 51.2 \\
\hline Females & 1,621 & 48.8 \\
\hline \multicolumn{3}{|l|}{ Overweight } \\
\hline$<85$ th percentile & 2,377 & 72 \\
\hline$>85$ th percentile & 938 & 28 \\
\hline \multicolumn{3}{|l|}{ Parents' education } \\
\hline$<$ High school & 477 & 21.8 \\
\hline High school graduate & 680 & 21.5 \\
\hline Some college & 561 & 17.1 \\
\hline College degree or higher & 1,597 & 39.6 \\
\hline \multicolumn{3}{|l|}{ Percentage of federal poverty level } \\
\hline $0-99$ & 351 & 16.2 \\
\hline $100-199$ & 591 & 21.2 \\
\hline $200-299$ & 410 & 13.2 \\
\hline$\geq 300$ & 1,963 & 49.4 \\
\hline \multicolumn{3}{|l|}{ Screen time (computer/television/video games) } \\
\hline$\leq 2 \mathrm{~h}$ & 653 & 19.1 \\
\hline$>2 \mathrm{~h}$ & 2,662 & 81 \\
\hline \multicolumn{3}{|l|}{ Physical activity (60 $\mathrm{min} /$ day of moderate activity) } \\
\hline$\geq 3$ days/week & 2,453 & 74 \\
\hline$<3$ days/week & 862 & 26 \\
\hline \multicolumn{3}{|l|}{ Breakfast consumption } \\
\hline Does not eat breakfast every day & 1,598 & 50.3 \\
\hline Does eat breakfast every day & 1,717 & 49.7 \\
\hline \multicolumn{3}{|l|}{ Family meals } \\
\hline Does not eat dinner with parent every night & 1,485 & 44.7 \\
\hline Does eat dinner with parent every night & 1,830 & 55.3 \\
\hline
\end{tabular}

${ }^{a}$ Weighted to represent population

Prevalence of overweight by sex and ethnicity

Approximately $34 \%$ of adolescent boys in California had BMIs greater than the 85th percentile for age and gender, compared with $22 \%$ of girls $\left(\chi^{2}=62.29, p<0.001\right.$; Table 4$)$. Latino adolescents $(37.5 \%)$ were more likely to be overweight than whites $(25 \%)$ or Asian Americans (16\%) $\left(\chi^{2}=91.5661, p<0.001\right)$. A higher percentage of adolescents between the ages of 12 and $13(32 \%)$ were overweight than ages 14 and $15(26 \%)$ or 16 and 17 $\left(27 \% ; \chi^{2}=10.10, p=0.05\right)$. Approximately $29 \%$ of adolescents who did not meet the APA's standard for physical activity were overweight, but $28 \%$ of adolescents who reported meeting the APA's standard also were overweight. Similarly, adolescents who did not meet the recommendation for sedentary activity (29\%) were more likely to be overweight than were adolescents who met this standard $(25.3 \%)\left(\chi^{2}=3.02, p=0.27\right)$.

Adolescents who did not eat breakfast daily were more likely to be overweight than were adolescents who ate breakfast every morning $\left(\chi^{2}=16.26, p<0.005\right)$. Parents' educational level also was related to overweight status in adolescents. The less education that a parent had attained, the more likely the adolescent was overweight; $38 \%$ of adolescents with parents with a high school education were overweight, whereas only $20 \%$ of adolescents with parents who had a college degree or higher were reported as overweight $\left(\chi^{2}=90.03, p<0.001\right)$. Family income level was also significant; income at the poverty level was more likely to be associated with overweight, compared with adolescents with family income of $300 \%$ of FPL or greater $\left(\chi^{2}=48.80, p<0.001\right.$; Table 4).

\section{Factors associated with overweight}

Multiple logistic regression was performed to assess the effect of a number of factors on the likelihood that respondents would report that they were overweight. The model contained 12 independent variables [sex, race (2 comparisons), meeting recommendation for physical activity, meeting recommendation for sedentary activity, age (2 comparisons), parents' educational level, and poverty level (3 comparisons), daily meal with parent or guardian, and breakfast consumption]. The full model containing all predictors was statistically significant $\left(F_{9,70}=13.08, p<\right.$ 0.001 ), indicating that the model could distinguish between respondents who reported and did not report being overweight. The model as a whole explained between $6.4 \%$ (Cox and Snell $\mathrm{R}^{2}$ ) and 9.4\% (Nagelkerke $\mathrm{R}^{2}$ ) of the variance in overweight status and correctly classified $74.4 \%$ of cases.

Only four of the independent variables (sex, age, parents' educational level, and breakfast consumption) made a unique statistically significant contribution to the model (Table 5A). The strongest predictor of an adolescent reporting himself or herself as overweight was gender, with an odds ratio of 2.03. Thus, male adolescents were almost twice as likely as female adolescents to report being overweight, once all other factors in the model were controlled for. The odds ratio of 0.76 for parents' educational level was less than 1 , indicating that as adult education level increased it was 0.76 times less likely that 
Table 2 Means of continuous variables ${ }^{\mathrm{a}}$

Continuous variable

\begin{tabular}{|c|c|c|c|c|c|c|}
\hline \multirow[b]{2}{*}{ Group } & \multicolumn{6}{|c|}{ Hours of sedentary activity } \\
\hline & Per day & Per weekday & Per weekend day & $\begin{array}{l}\text { Days with } 60 \text { min of } \\
\text { moderate physical activity }\end{array}$ & Days eats breakfast & Days eats dinner with parents \\
\hline \multicolumn{7}{|l|}{ Overall } \\
\hline Mean & 3.82 & 3.35 & 4.29 & 3.90 & 4.99 & 5.43 \\
\hline $\mathrm{SE}$ & 0.67 & 0.073 & 0.083 & 0.54 & 0.063 & 0.062 \\
\hline $\mathrm{CI}$ & $3.69-3.96$ & $3.21-3.50$ & $4.13-4.46$ & $3.79-4.00$ & $4.87-5.12$ & $5.31-5.60$ \\
\hline \multicolumn{7}{|l|}{ Whites } \\
\hline Mean & 3.70 & & & 4.12 & 5.05 & 5.58 \\
\hline SE & 0.071 & & & 0.066 & 0.067 & 0.069 \\
\hline $\mathrm{CI}$ & $3.56-3.84$ & & & $3.99-4.25$ & $4.92-5.19$ & $5.44-5.72$ \\
\hline \multicolumn{7}{|l|}{ Latinos } \\
\hline Mean & 3.72 & & & 3.76 & 4.80 & 5.04 \\
\hline SE & 0.119 & & & 0.096 & 0.138 & 0.112 \\
\hline $\mathrm{CI}$ & $3.49-3.96$ & & & $3.56-3.95$ & $4.53-5.08$ & $4.82-5.26$ \\
\hline \multicolumn{7}{|c|}{ Asian Americans } \\
\hline Mean & 4.54 & & & 3.43 & 5.26 & 5.88 \\
\hline SE & 0.183 & & & 0.170 & 0.166 & 0.069 \\
\hline $\mathrm{CI}$ & $4.18-4.91$ & & & $3.09-3.76$ & $4.93-5.60$ & $5.44-5.72$ \\
\hline \multicolumn{7}{|c|}{ White girls } \\
\hline Mean & 3.47 & & & 3.73 & 4.92 & 5.45 \\
\hline $\mathrm{SE}$ & 0.086 & & & 0.091 & 0.112 & 0.097 \\
\hline $\mathrm{CI}$ & $3.30-3.64$ & & & $3.55-3.91$ & $4.70-5.15$ & $5.26-5.64$ \\
\hline \multicolumn{7}{|c|}{ Latina girls } \\
\hline Mean & 3.89 & & & 3.45 & 4.60 & 4.63 \\
\hline SE & 0.162 & & & 0.133 & 0.191 & 0.183 \\
\hline $\mathrm{CI}$ & $3.56-4.21$ & & & $3.18-3.71$ & $4.22-4.98$ & $4.30-4.99$ \\
\hline \multicolumn{7}{|c|}{ Asian American girls } \\
\hline Mean & 4.11 & & & 3.31 & 5.40 & 5.90 \\
\hline SE & 0.2406 & & & 0.290 & 0.290 & 0.235 \\
\hline $\mathrm{CI}$ & $3.63-4.59$ & & & $2.73-3.89$ & $4.80-5.94$ & $5.43-6.40$ \\
\hline \multicolumn{7}{|c|}{ White boys } \\
\hline Mean & 3.92 & & & 4.50 & 5.18 & 5.70 \\
\hline $\mathrm{SE}$ & 0.095 & & & 0.081 & 0.101 & 0.085 \\
\hline $\mathrm{CI}$ & $3.73-4.11$ & & & $4.33-4.65$ & $4.98-5.38$ & $5.53-5.87$ \\
\hline \multicolumn{7}{|c|}{ Latino boys } \\
\hline Mean & 3.60 & & & 4.05 & 5.01 & 5.44 \\
\hline SE & 0.162 & & & 0.147 & 0.168 & 0.161 \\
\hline $\mathrm{CI}$ & $3.25-3.89$ & & & $3.80-4.35$ & $4.67-5.34$ & $5.12-5.76$ \\
\hline \multicolumn{7}{|c|}{ Asian American boys } \\
\hline Mean & 4.94 & & & 3.53 & 5.20 & 5.86 \\
\hline $\mathrm{SE}$ & 0.285 & & & 0.240 & 0.231 & 0.217 \\
\hline CI & $4.37-5.50$ & & & $3.06-4.00$ & $4.71-5.63$ & $5.43-6.29$ \\
\hline
\end{tabular}

${ }^{\text {a }}$ Values are mean, standard error (SE, weighted), and 95\% confidence interval (CI) 
Table 3 Demographics of the study by gender and race*. *Percentages in bold are weighted and represent population estimation

\begin{tabular}{|c|c|c|c|c|c|c|}
\hline \multirow[b]{2}{*}{ Characteristics } & \multicolumn{3}{|c|}{ Girls $(n=1,621,48.9 \%)$} & \multicolumn{3}{|c|}{ Boys $(\mathrm{n}=1,694,51.1 \%)$} \\
\hline & $\begin{array}{l}\text { White } \\
(\mathrm{n}=1,070)\end{array}$ & $\begin{array}{l}\text { Latina } \\
(\mathrm{n}=407)\end{array}$ & $\begin{array}{l}\text { Asian American } \\
(\mathrm{n}=144)\end{array}$ & $\begin{array}{l}\text { White } \\
(\mathrm{n}=1,065)\end{array}$ & $\begin{array}{l}\text { Latino } \\
(\mathrm{n}=420)\end{array}$ & $\begin{array}{l}\text { Asian American } \\
(\mathrm{n}=209)\end{array}$ \\
\hline \multicolumn{7}{|l|}{ Age, years } \\
\hline $12-13(\mathrm{n}=1,113,33.6 \%)$ & $\begin{array}{l}316(30 \%) \\
(28 \%)\end{array}$ & $\begin{array}{l}159(39 \%) \\
(36 \%)\end{array}$ & $\begin{array}{l}53(37 \%) \\
(31 \%)\end{array}$ & $\begin{array}{l}350(33 \%) \\
(29 \%)\end{array}$ & $\begin{array}{l}169(40 \%) \\
(39 \%)\end{array}$ & $\begin{array}{l}66(32 \%) \\
(29 \%)\end{array}$ \\
\hline $14-15(n=1,172,35.4 \%)$ & $\begin{array}{l}383(36 \%) \\
(38 \%)\end{array}$ & $\begin{array}{l}136(33 \%) \\
(34 \%)\end{array}$ & $\begin{array}{l}47(33 \%) \\
(35 \%)\end{array}$ & $\begin{array}{l}373(35 \%) \\
(34 \%)\end{array}$ & $\begin{array}{l}157(37 \%) \\
(36 \%)\end{array}$ & $\begin{array}{l}76(36 \%) \\
(35 \%)\end{array}$ \\
\hline $16-17(\mathrm{n}=1,030,31.1 \%)$ & $\begin{array}{l}371(35 \%) \\
(34 \%)\end{array}$ & $\begin{array}{l}112(28 \%) \\
(31 \%)\end{array}$ & $\begin{array}{l}44(31 \%) \\
(34 \%)\end{array}$ & $\begin{array}{l}342(32 \%) \\
(37 \%)\end{array}$ & $\begin{array}{l}94(22 \%) \\
(25 \%)\end{array}$ & $\begin{array}{l}67(32 \%) \\
(36 \%)\end{array}$ \\
\hline \multicolumn{7}{|l|}{ Parents' education } \\
\hline$>$ High school $(\mathrm{n}=477,14.4 \%)$ & $\begin{array}{l}43(4 \%) \\
(5 \%)\end{array}$ & $\begin{array}{l}201(49 \%) \\
(54 \%)\end{array}$ & $\begin{array}{l}13(9 \%) \\
(15 \%)\end{array}$ & $\begin{array}{l}27(3 \%) \\
(4 \%)\end{array}$ & $\begin{array}{l}181(43 \%) \\
(50 \%)\end{array}$ & $\begin{array}{l}12(6 \%) \\
(3 \%)\end{array}$ \\
\hline High school or equivalent $(n=680,20.5 \%)$ & $\begin{array}{l}227(21 \%) \\
(23 \%)\end{array}$ & $\begin{array}{l}93(23 \%) \\
(22 \%)\end{array}$ & $\begin{array}{l}24(17 \%) \\
(19 \%)\end{array}$ & $\begin{array}{l}195(18 \%) \\
(21 \%)\end{array}$ & $\begin{array}{l}100(24 \%) \\
(22 \%)\end{array}$ & $\begin{array}{l}41(20 \%) \\
(20 \%)\end{array}$ \\
\hline Some college $(n=561,16.9 \%)$ & $\begin{array}{l}229(21 \%) \\
(24 \%)\end{array}$ & $\begin{array}{l}41(10 \%) \\
(9 \%)\end{array}$ & $\begin{array}{l}15(10 \%) \\
(12 \%)\end{array}$ & $\begin{array}{l}196(18 \%) \\
(21 \%)\end{array}$ & $\begin{array}{l}58(14 \%) \\
(12 \%)\end{array}$ & $\begin{array}{l}22(11 \%) \\
(17 \%)\end{array}$ \\
\hline College degree or higher $(\mathrm{n}=1,597,48.2 \%)$ & $\begin{array}{l}571(53 \%) \\
(48 \%)\end{array}$ & $\begin{array}{l}72(18 \%) \\
(15 \%)\end{array}$ & $\begin{array}{l}92(64 \%) \\
(54 \%)\end{array}$ & $\begin{array}{l}647(61 \%) \\
(54 \%)\end{array}$ & $\begin{array}{l}81(19 \%) \\
(16 \%)\end{array}$ & $\begin{array}{l}134(64 \%) \\
(59 \%)\end{array}$ \\
\hline \multicolumn{7}{|l|}{ Percentage of federal poverty level } \\
\hline $0-99(\mathrm{n}=351=10.6 \%)$ & $\begin{array}{l}49(5 \%) \\
(5 \%)\end{array}$ & $\begin{array}{l}111(27 \%) \\
(33 \%)\end{array}$ & $\begin{array}{l}19(13 \%) \\
(23 \%)\end{array}$ & $\begin{array}{l}34(3 \%) \\
(4 \%)\end{array}$ & $\begin{array}{l}113(27 \%) \\
(32 \%)\end{array}$ & $\begin{array}{l}25(12 \%) \\
(13 \%)\end{array}$ \\
\hline $100-199(\mathrm{n}=591,17.8 \%)$ & $\begin{array}{l}118(11 \%) \\
(13 \%)\end{array}$ & $\begin{array}{l}145(36 \%) \\
(37 \%)\end{array}$ & $\begin{array}{l}24(17 \%) \\
(19 \%)\end{array}$ & $\begin{array}{l}121(11 \%) \\
(12 \%)\end{array}$ & $\begin{array}{l}149(36 \%) \\
(34 \%)\end{array}$ & $\begin{array}{l}34(16 \%) \\
(15 \%)\end{array}$ \\
\hline $200-299(n=410,12.4 \%)$ & $\begin{array}{l}149(14 \%) \\
(16 \%)\end{array}$ & $\begin{array}{l}44(11 \%) \\
(9 \%)\end{array}$ & $\begin{array}{l}20(14 \%) \\
(18 \%)\end{array}$ & $\begin{array}{l}122(12 \%) \\
(12 \%)\end{array}$ & $\begin{array}{l}52(12 \%) \\
(12 \%)\end{array}$ & $\begin{array}{l}23(11 \%) \\
(17 \%)\end{array}$ \\
\hline$>300(\mathrm{n}=1,963,59.2 \%)$ & $\begin{array}{l}754(71 \%) \\
(67 \%)\end{array}$ & $\begin{array}{l}107(26 \%) \\
(20 \%)\end{array}$ & $\begin{array}{l}81(56 \%) \\
(40 \%)\end{array}$ & $\begin{array}{l}788(74 \%) \\
(72 \%)\end{array}$ & $\begin{array}{l}106(25 \%) \\
(22 \%)\end{array}$ & $\begin{array}{l}127(61 \%) \\
(54 \%)\end{array}$ \\
\hline \multicolumn{7}{|l|}{ Screen time (computer/television) } \\
\hline$\leq 2 \mathrm{~h} /$ day $(\mathrm{n}=653,19.7 \%)$ & $\begin{array}{l}248(23 \%) \\
(23 \%)\end{array}$ & $\begin{array}{l}77(19 \%) \\
(17 \%)\end{array}$ & $\begin{array}{l}25(17 \%) \\
(13 \%)\end{array}$ & $\begin{array}{l}193(18 \%) \\
(16 \%)\end{array}$ & $\begin{array}{l}93(22 \%) \\
(28 \%)\end{array}$ & $\begin{array}{l}17(8 \%) \\
(9 \%)\end{array}$ \\
\hline$>2$ h/day $(n=2,662,80.3 \%)$ & $\begin{array}{l}822(77 \%) \\
(77 \%)\end{array}$ & $\begin{array}{l}330(81 \%) \\
(83 \%)\end{array}$ & $\begin{array}{l}119(83 \%) \\
(87 \%)\end{array}$ & $\begin{array}{l}872(82 \%) \\
(84 \%)\end{array}$ & $\begin{array}{l}327(78 \%) \\
(72 \%)\end{array}$ & $\begin{array}{l}192(92 \%) \\
(91 \%)\end{array}$ \\
\hline \multicolumn{7}{|c|}{ Physical activity (60 min of moderate physical activity/day) } \\
\hline$>3$ days/week $(\mathrm{n}=2,487,75 \%)$ & $\begin{array}{l}804(75 \%) \\
(72 \%)\end{array}$ & $\begin{array}{l}264(65 \%) \\
(66 \%)\end{array}$ & $\begin{array}{l}88(61 \%) \\
(57 \%)\end{array}$ & $\begin{array}{l}874(82 \%) \\
(84 \%)\end{array}$ & $\begin{array}{l}326(78 \%) \\
(74 \%)\end{array}$ & $\begin{array}{l}131(63 \%) \\
(63 \%)\end{array}$ \\
\hline$<3$ days/week $(\mathrm{n}=828,25 \%)$ & $\begin{array}{l}266(25 \%) \\
(28 \%)\end{array}$ & $\begin{array}{l}143(35 \%) \\
(34 \%)\end{array}$ & $\begin{array}{l}56(39 \%) \\
(43 \%)\end{array}$ & $\begin{array}{l}191(18 \%) \\
(16 \%)\end{array}$ & $\begin{array}{l}94(22 \%) \\
(26 \%)\end{array}$ & $\begin{array}{l}78(37 \%) \\
(37 \%)\end{array}$ \\
\hline \multicolumn{7}{|l|}{ Breakfast consumption } \\
\hline $0-6$ days/week $(n=1,598,48.2 \%)$ & $\begin{array}{l}557(52 \%) \\
(53 \%)\end{array}$ & $\begin{array}{l}220(54 \%) \\
(52 \%)\end{array}$ & $\begin{array}{l}59(41 \%) \\
(44 \%)\end{array}$ & $\begin{array}{l}464(44 \%) \\
(46 \%)\end{array}$ & $\begin{array}{l}205(49 \%) \\
(52 \%)\end{array}$ & $\begin{array}{l}93(45 \%) \\
(53 \%)\end{array}$ \\
\hline 7 days/week $(\mathrm{n}=1,717,51.8 \%)$ & $\begin{array}{l}513(48 \%) \\
(47 \%)\end{array}$ & $\begin{array}{l}187(46 \%) \\
(48 \%)\end{array}$ & $\begin{array}{l}85(59 \%) \\
(56 \%)\end{array}$ & $\begin{array}{l}601(56 \%) \\
(54 \%)\end{array}$ & $\begin{array}{l}215(51 \%) \\
(48 \%)\end{array}$ & $\begin{array}{l}116(56 \%) \\
(47 \%)\end{array}$ \\
\hline \multicolumn{7}{|l|}{ Family meals } \\
\hline $0-6$ days/week $(n=1,485,44.8 \%)$ & $\begin{array}{l}522(49 \%) \\
(48 \%)\end{array}$ & $\begin{array}{l}197(48 \%) \\
(55 \%)\end{array}$ & $\begin{array}{l}49(34 \%) \\
(29 \%)\end{array}$ & $\begin{array}{l}475(45 \%) \\
(44 \%)\end{array}$ & $\begin{array}{l}169(40 \%) \\
(42 \%)\end{array}$ & $\begin{array}{l}76(36 \%) \\
(34 \%)\end{array}$ \\
\hline 7 days/week $(\mathrm{n}=1,830,55.2 \%)$ & $\begin{array}{l}548(51 \%) \\
(52 \%)\end{array}$ & $\begin{array}{l}213(52 \%) \\
(45 \%)\end{array}$ & $\begin{array}{l}95(66 \%) \\
(71 \%)\end{array}$ & $\begin{array}{l}590(55 \%) \\
(56 \%)\end{array}$ & $\begin{array}{l}251(60 \%) \\
(58 \%)\end{array}$ & $\begin{array}{l}133(64 \%) \\
(66 \%)\end{array}$ \\
\hline
\end{tabular}


Table 3 (continued)

\begin{tabular}{|c|c|c|c|c|c|c|}
\hline \multirow[b]{2}{*}{ Characteristics } & \multicolumn{3}{|c|}{ Girls $(n=1,621,48.9 \%)$} & \multicolumn{3}{|c|}{ Boys $(\mathrm{n}=1,694,51.1 \%)$} \\
\hline & $\begin{array}{l}\text { White } \\
(\mathrm{n}=1,070)\end{array}$ & $\begin{array}{l}\text { Latina } \\
(\mathrm{n}=407)\end{array}$ & $\begin{array}{l}\text { Asian American } \\
(\mathrm{n}=144)\end{array}$ & $\begin{array}{l}\text { White } \\
(\mathrm{n}=1,065)\end{array}$ & $\begin{array}{l}\text { Latino } \\
(\mathrm{n}=420)\end{array}$ & $\begin{array}{l}\text { Asian American } \\
(\mathrm{n}=209)\end{array}$ \\
\hline \multicolumn{7}{|l|}{ Body mass index } \\
\hline$<85$ th percentile $(n=2,464,74.4 \%)$ & $\begin{array}{l}875(82 \%) \\
(82 \%)\end{array}$ & $\begin{array}{l}272(67 \%) \\
(66 \%)\end{array}$ & $\begin{array}{l}138(96 \%) \\
(96 \%)\end{array}$ & $\begin{array}{l}787(74 \%) \\
(69 \%)\end{array}$ & $\begin{array}{l}247(59 \%) \\
(59 \%)\end{array}$ & $\begin{array}{l}148(71 \%) \\
(73 \%)\end{array}$ \\
\hline$>85$ th percentile $(n=850,25.6 \%)$ & $\begin{array}{l}197(18 \%) \\
(18 \%)\end{array}$ & $\begin{array}{l}135(33 \%) \\
(34 \%)\end{array}$ & $\begin{array}{l}6(4 \%) \\
(4 \%)\end{array}$ & $\begin{array}{l}278(26 \%) \\
(31 \%)\end{array}$ & $\begin{array}{l}173(41 \%) \\
(41 \%)\end{array}$ & $\begin{array}{l}61(29 \%) \\
(27 \%)\end{array}$ \\
\hline
\end{tabular}

the adolescent would report being overweight, once the other factors in the model were controlled for.

Multivariate logistic regression by ethnicity and gender

A multiple logistic regression model (Table 5B) was formulated, with gender and ethnicity controlled for, to examine factors contributing to overweight. White girls were 2.73 times $(p=0.008)$ more likely to be overweight if they had a low socioeconomic status (100\%-199\% FPL compared with $>300 \%$ FPL). Latina girls were seven times more likely to be overweight if their parents had less than a high school education $(p=0.006)$. Asian American girls were 2.97 times less likely to be overweight as their parents' educational level increased $(p=0.01)$. Two factors contributed to overweight in white boys: higher screen time $(p=0.001)$ and lower parents' educational level $(p=0.004)$. Latino boys also were 2.6 times more likely to be overweight if their parents had only a high school education. Asian American boys had no factors in the model that were associated with an increased risk of overweight. The differences found among ethnicities and genders seem unique except for parents' educational level, which contributed to overweight in Latino boys and girls as well as Asian American girls and white boys.

\section{Univariate logistic regression}

According to univariate logistic regression (Table 6), boys were 1.86 times as likely as girls to be overweight ( $p<$ 0.001). Latinos were more likely to be overweight than whites (1.81, $p<0.001)$, whereas Asian Americans were less likely to be overweight than whites $(0.58, p<0.001)$. Neither meeting the recommendation for physical activity set forth by the APA or meeting the screen time recommendations were statistically significant contributors to adolescents' being overweight (Table 6). Daily breakfast consumption had an odds ratio of 0.73 , indicating that the likelihood of being overweight decreased as the frequency of eating breakfast increased $(p=0.006)$. When the three age groups (12-13, 14-15, and 16-17 years old) were compared, with the 16 to 17 year olds used as the reference group, no significant differences were noted. When the percentage of FPL (0-99, 100-199, 200-299, >300) were compared, with $>300 \%$ FPL used as the reference group, adolescents in the lower income groups (0\%-99\% FPL and $100 \%-199 \%$ FPL) were 1.89 times and 1.69 times, respectively, more likely to be overweight than were adolescents in the $>300 \%$ FPL group $(p<0.001)$.

\section{Discussion}

Although factors related to obesity in adolescents have been examined in several studies, this study is one of the first in which ethnic and gender differences in obesity in California were explored. Our findings indicated ethnic and gender differences in overweight in California adolescents. Adolescent obesity is a multifactorial condition influenced by both modifiable and nonmodifiable factors (Perry et al. 2004). The ecological systems theory emphasizes the importance of considering the individual as well as the context within which the adolescent is embedded. Behavioral choices are not made in a vacuum or without the broader social environment including the family, community, and broader society being considered. Our study showed Latino boys (41\%) were more likely than any other group to be overweight followed by Latina girls (34\%), white boys (31.2\%), Asian American boys (27\%), white girls (18\%), and Asian American girls (4\%). National statistics (National Health and Nutrition Examination Survey, 2003-2004) indicate that the prevalence in overweight was $22.8 \%$ (male) and $10.4 \%$ (female). Asian American adolescents compared with $26.5 \%$ (male) and $22.2 \%$ (female) in white adolescents, whereas Latinos had the highest rates of overweight with $37.3 \%$ (male) and 31.1\% (female) (Gordon-Larsen et al. 2003). Although Asian Americans have the lowest prevalence of overweight, studies have indicated different cutoff points for Asian Americans because of their higher risk of cardiovascular disease at low BMIs (Freedman et al. 2008). 
Table 4 Cross tabulations based on overweight status ${ }^{\mathrm{a}}$

\begin{tabular}{|c|c|c|c|}
\hline \multirow[b]{2}{*}{ Independent variable } & \multicolumn{2}{|l|}{ Dependent variable } & \multirow[b]{2}{*}{$p$ (from $\chi^{2}$ Test $)$} \\
\hline & Not overweight, $<85$ th percentile & Overweight, $>85$ th percentile & \\
\hline Gender & & & $<0.001$ \\
\hline Male & $\begin{array}{l}1,182(70 \%) \\
(66 \%, 0.017,0.625-0.691)\end{array}$ & $\begin{array}{l}512(30 \%) \\
(34 \%, 0.017,0.309-0.375)\end{array}$ & \\
\hline Female & $\begin{array}{l}1,283(79 \%) \\
(78 \%, 0.013,0.755-0.807)\end{array}$ & $\begin{array}{l}338(21 \%) \\
(22 \%, 0.013,0.193-0.245)\end{array}$ & \\
\hline Ethnicity & & & $<0.001$ \\
\hline White & $\begin{array}{l}1,660(78 \%) \\
(75.1 \%, 0.013,0.725-0.776)\end{array}$ & $\begin{array}{l}475(22 \%) \\
(25 \%, 0.013,0.224-0.276)\end{array}$ & \\
\hline Latino & $\begin{array}{l}519(63 \%) \\
(63 \%, 0.022,0.581-0.667)\end{array}$ & $\begin{array}{l}308(37 \%) \\
(37.5 \%, 0.022,0.333-0.419)\end{array}$ & \\
\hline Asian American & $\begin{array}{l}286(81 \%) \\
(84 \%, 0.025,0.784-0.883)\end{array}$ & $\begin{array}{l}67(19 \%) \\
(16 \%, 0.025,0.117-0.216)\end{array}$ & \\
\hline Age, years & & & 0.05 \\
\hline $12-13$ & $\begin{array}{l}804(72 \%) \\
(68.2 \%, 0.019,0.643-0.720)\end{array}$ & $\begin{array}{l}309(28 \%) \\
(32 \%, 0.020,0.280-0.357)\end{array}$ & \\
\hline $14-15$ & $\begin{array}{l}865(74 \%) \\
(74 \%, 0.015,0.708-0.768)\end{array}$ & $\begin{array}{l}307(26 \%) \\
(26 \%, 0.015,0.232-0.292)\end{array}$ & \\
\hline $16-17$ & $\begin{array}{l}796(77 \%) \\
(73.2 \%, 0.018,0.695-0.767)\end{array}$ & $\begin{array}{l}234(23 \%) \\
(27 \%, 0.018,0.233-0.305)\end{array}$ & \\
\hline Physical activity & & & 0.77 \\
\hline Meets standard $>3$ days/week & $\begin{array}{l}1,873(75.3 \%) \\
(72 \%, 0.013,0.700-0.750)\end{array}$ & $\begin{array}{l}614(24.7 \%) \\
(28 \%, 0.013,0.255-0.305)\end{array}$ & \\
\hline Does not meet standard & $\begin{array}{l}592(72 \%) \\
(71.3 \%, 0.02,0.670-0.750)\end{array}$ & $\begin{array}{l}236(29 \%) \\
(29 \%, 0.022,0.250-0.331)\end{array}$ & \\
\hline Sedentary activity & & & 0.27 \\
\hline Meets standard $<2 \mathrm{~h} /$ day & $\begin{array}{l}510(78 \%) \\
(75 \%, 0.027,0.691-0.796)\end{array}$ & $\begin{array}{l}143(22 \%) \\
(25.3 \%, 0.027,0.204-0.310)\end{array}$ & \\
\hline Does not meet standard & $\begin{array}{l}1,955(73 \%) \\
(71 \%, 0.012,0.689-0.735)\end{array}$ & $\begin{array}{l}707(27 \%) \\
(29 \%, 0.012,0.265-0.311)\end{array}$ & \\
\hline Breakfast consumption & & & $<0.005$ \\
\hline Yes & $\begin{array}{l}1,340(78 \%) \\
(75.1 \%, 0.014,0.721-0.778)\end{array}$ & $\begin{array}{l}377(22 \%) \\
(25 \%, 0.014,0.222-0.279)\end{array}$ & \\
\hline No & $\begin{array}{l}1,125(70 \%) \\
(69 \%, 0.016,0.656-0.718)\end{array}$ & $\begin{array}{l}473(30 \%) \\
(31.2 \%, 0.016,0.282-0.344)\end{array}$ & \\
\hline Family meals & & & 0.33 \\
\hline Yes & $\begin{array}{l}1,354(74 \%) \\
(73 \%, 0.013,0.701-0.754)\end{array}$ & $\begin{array}{l}476(26 \%) \\
(27 \%, 0.013,0.246-0.299)\end{array}$ & \\
\hline No & $\begin{array}{l}1,111(75 \%) \\
(71 \%, 0.020,0.674-0.739)\end{array}$ & $\begin{array}{l}374(25 \%) \\
(29.2 \%, 0.016,0.261-0.326)\end{array}$ & \\
\hline Parents' educational level & & & $<0.001$ \\
\hline$<$ High school & $\begin{array}{l}292(61 \%) \\
(62 \%, 0.028,0.568-0.678)\end{array}$ & $\begin{array}{l}185(39 \%) \\
(38 \%, 0.028,0.322-0.432)\end{array}$ & \\
\hline High school graduate & $\begin{array}{l}460(68 \%) \\
(66 \%, 0.023,0.610-0.701)\end{array}$ & $\begin{array}{l}220(32 \%) \\
(34 \%, 0.023,0.299-0.390)\end{array}$ & \\
\hline Some college & $\begin{array}{l}419(75 \%) \\
(73 \%, 0.03,0.662-0.781)\end{array}$ & $\begin{array}{l}142(25 \%) \\
(27 \%, 0.03,0.219-0.338)\end{array}$ & \\
\hline College degree or higher & $\begin{array}{l}1,294(81 \%) \\
(80.1 \%, 0.011,0.778-0.823)\end{array}$ & $\begin{array}{l}303(19 \%) \\
(20 \%, 0.011,0.177-0.222)\end{array}$ & \\
\hline$\%$ of federal poverty level & & & $<0.001$ \\
\hline $0-99$ & $\begin{array}{l}227(65 \%) \\
(64 \%, 0.036,0.564-0.706)\end{array}$ & $\begin{array}{l}124(35 \%) \\
(36 \%, 0.036,0.294-0.436)\end{array}$ & \\
\hline $100-199$ & $378(64 \%)$ & $213(36 \%)$ & \\
\hline
\end{tabular}


Table 4 (continued)

\begin{tabular}{lll}
\hline & Dependent variable & \\
\cline { 2 - 3 } Independent variable & Not overweight, $<85$ th percentile & Overweight, $>85$ th percentile \\
\hline \multirow{3}{*}{$200-299$} & $(66 \%, 0.254,0.612-0.713)$ & $(34 \%, 0.025,0.288-0.388)$ \\
& $303(73.9 \%)$ & $107(26.1 \%)$ \\
& $(72 \%, 0.032,0.648-0.776)$ & $(28 \%, 0.032,0.224-0.352)$ \\
& $1,557(79 \%)$ & $406(21 \%)$ \\
& $(77 \%, 0.013,0.744-0.794)$ & $(23 \%, 0.013,0.206-0.256)$ \\
\hline
\end{tabular}

${ }^{a}$ Numbers in bold are weighted (percentage, standard error, 95\% confidence limits)

The prevalence of being at risk for overweight and being overweight are higher among 6 to 11 year olds than among 12 to 19 year olds (Ogden et al. 2006). This finding was confirmed by the results of our study, with $32 \%$ of children in the 12- to 13-year-old group being overweight compared with $26 \%$ of the 14 - to 15 -year-old group $(p=0.05)$. Similar to 14 and 15 year olds, $27 \%$ of 16 and 17 year olds were overweight; therefore, overweight prevention should start in early childhood.

Our study results indicate that white girls were at greater risk of being overweight if they had a lower household income. Published reports indicate that socioeconomic status is a factor that contributes to overweight status, especially in US whites, but also to a lesser degree in minorities (Gordon-Larsen et al. 2003; Scharoun-Lee et al. 2009; Zhang and Wang 2004). The literature also suggests that socioeconomic status has a different effect on overweight in white (high socioeconomic status = low BMI in whites) compared with African American (high socioeconomic status $=$ high $\mathrm{BMI})$. Our results support the published results in that white girls were the only group in which low socioeconomic status $(\%$ FPL <199) made a statistically significant contribution to overweight. Environmental factors associated with low socioeconomic status, including increased access to fast food and convenience stores rather than access to grocery stores, may contribute. Unsafe neighborhoods or limited exercise facilities may also contribute to our finding. However, the reason socioeconomic status affects the risk of being overweight only in white girls remains unclear. Researchers should examine the potential mechanisms by which socioeconomic status affects risk of overweight in white girls.

Several of our groups had a higher risk of being overweight if their parents had low educational levels. Parent educational level has also been associated with overweight status in children (Chen 2009; Parizkova 2008). For Latino boys and girls as well as Asian American girls and white boys, this relationship was found to hold true, but it was not a factor in the other ethnic/gender groups. Future research should look into the variations and possible explanations for the influence of parents' educational level on overweight risk among various ethnicities and different genders. For white boys, screen time contributed to the overweight status along with the parents' educational level.

Breakfast was not a significant factor for any ethnic/ gender group, which was an unexpected finding. Less desirable meal patterns such as skipping meals have also been reported to contribute to increased risk of being overweight (Berkey et al. 2003). According to Sinha and King (2008), the habit of skipping breakfast and overeating dinner increases the risk of overweight. In our study, breakfast consumption was associated with increased risk of overweight in some gender/ethnic groups and was a statistically significant factor for all adolescents in general. In several cross-sectional studies, mainly in white girls, researchers have found an inverse relationship between eating breakfast and BMI (Timlin et al. 2008). Boutelle et al.(2002) report an ethnically diverse sample, but analyzed data only by gender. Limited research exists related to difference in breakfast consumption and the effect on BMI in an ethnically diverse sample (Pearson et al. 2009). It is unclear why not eating breakfast was not a risk factor based on gender and ethnicity, but as rates of breakfast consumption decrease throughout adolescence and into adulthood, the impact of regular breakfast consumption may be an important variable to consider. This area of ethnic differences and skipped meals has not been researched, and further research should explore ethnic and gender differences in breakfast consumption and the relationship to overweight status.

In our study, frequency of family meals was not a significant contributor to overweight in Latino, Asian American, or white California adolescents. Frequency of meals may not be a factor because of the older age of the sample (early and late adolescents rather than preschool and school-age children) and their increasing independence and autonomy around food choices and meal times. The literature related to frequency of family meals and weight status is controversial. Fulkerson et al. (2007) report middle school girls have a significant risk of being overweight if they do not eat regular family meals. Gable et al. (2007) 
Table 5 Multiple logistic regression

Overall sample variable

A. Predicting likelihood of overweight adolescents

Physical activity
Screen time
Breakfast consumption
Family meals
Gender
Age
Parents' educational level
Poverty level
Race

Group/significant factors

B. Summary table by gender and ethnicity

White girls $(n=1,070)$

$\%$ Federal poverty level

$0-99$ vs $\geq 300$

$100-199$ vs $\geq 300$

200-299 vs $\geq 300$

Latina girls $(n=407)$

Parents' educational level $<$ high school vs college or more

Asian American girls $(\mathrm{n}=144)$

Parents' educational level

White boys $(n=1,065)$

Screen time

Parents' educational level $<$ high school vs college or more

High school/diploma vs college or more

Latino boys $(\mathrm{n}=420)$

Parents' educational level high school/diploma vs college or more
Odds ratio

$\begin{array}{llll}1.03 & 0.15 & 0.19 & 0.85 \\ 1.27 & 0.20 & 1.54 & 0.13 \\ 0.75 & 0.09 & -2.42 & 0.02 \\ 0.93 & 0.11 & -0.58 & 0.6 \\ 2.03 & 0.24 & 6.02 & <0.001 \\ 0.87 & 0.06 & -2.01 & 0.048 \\ 0.76 & 0.05 & -4.53 & <0.001 \\ 0.90 & 0.07 & -1.35 & 0.18 \\ 0.83 & 0.08 & -1.92 & 0.06\end{array}$

$0.589-0.950$

$1.609-2.570$

$0.766-0.999$

$0.669-0.855$
SE $\quad t$

0.08
$95 \% \mathrm{CI}$

\begin{tabular}{lllll}
0.68 & 0.08 & -3.11 & 0.003 & $0.528-0.869$ \\
2.45 & 1.08 & 2.02 & 0.047 & $1.010-5.900$ \\
2.73 & 1.01 & 2.72 & 0.008 & $1.310-5.700$ \\
1.56 & 0.50 & 1.40 & 0.73 & $0.436-1.790$ \\
0.13 & & & & \\
& 0.09 & -2.85 & 0.006 & $0.031-0.537$ \\
2.97 & 1.23 & 2.62 & 0.01 & $1.300-6.780$ \\
2.28 & & & & \\
0.26 & 0.56 & 3.38 & 0.001 & $1.405-3.710$ \\
2.10 & 0.17 & -2.06 & 0.04 & $0.070-0.950$ \\
& 0.53 & 2.94 & 0.004 & $1.270-3.470$ \\
2.60 & & & & \\
& 1.11 & 2.24 & 0.03 & $1.110-6.080$ \\
\hline
\end{tabular}

assessed family meal frequency in school-age children and found a significant inverse association between frequency of family meals and overweight status. The significance of the frequency of family meals seems to vary with gender (Neumark-Sztainer et al. 2007), highlighting the need for additional research in this area.

Asian American boys had no specific factors that contributed significantly to overweight status. It is unclear why sedentary activity, which was highest in Asian American boys ( $91 \%$ had $>2 \mathrm{~h} /$ day), was not a significant contributor to overweight status in this group. Asian American boys and girls were also the groups that were least likely to achieve $3 \mathrm{~h}$ per week of physical activity. In contrast, two behaviors that have been linked to more healthy weight status were more prevalent in Asian American girls: eating breakfast and sharing family meals on a daily basis. Possibly regular meals shared with the family improved this group's nutritional status, which is known to be protective.
Several factors contributed to overweight status in white boys, including screen time and educational level of their parents. As indicated by the principle of thermodynamics (energy in must equal energy out), physical activity, sedentary activity, and nutritional factors also play an important role in weight status (Lustig 2006; CDC 2009b). Interestingly, screen time (sedentary activity) contributed to overweight status only in white boys, who spent an average of $3.92 \mathrm{~h}$ /day (compared with national averages of $4.6 \mathrm{~h} /$ day viewing) watching television or playing on the computer or with video games. Despite Asian American boys averaging $4.94 \mathrm{~h}$ of sedentary activity, more than twice the APA's recommendation of less than $2 \mathrm{~h}$ daily, and being physically active for $60 \mathrm{~min}$ or more for only 3.53 days, minimally meeting the APA's recommendation of $60 \mathrm{~min}$ of moderate physical activity 3 or more days per week, neither of these factors contributed significantly to overweight in this group. Several studies have indicated that the prevalence of adequate physical activity is particularly low among His- 
Table 6 Univariate logistic regression, with overweight as the dependent variable

\begin{tabular}{|c|c|c|c|c|}
\hline Independent variable & Odds ratio & $p^{*}$ & Lower bound & Upper bound \\
\hline Male vs female & 1.86 & $<0.001$ & 1.490 & 2.300 \\
\hline \multicolumn{5}{|l|}{ Ethnicity } \\
\hline White vs Latino & 1.81 & $<0.001$ & 1.418 & 2.310 \\
\hline White vs Asian American & 0.58 & 0.01 & 0.382 & 0.873 \\
\hline Physical activity recommendations met & 1.04 & 0.08 & & \\
\hline Screen time recommendations met & 1.20 & 0.27 & & \\
\hline Breakfast consumption & 0.73 & 0.006 & 0.588 & 0.910 \\
\hline Family meals & 0.90 & 0.33 & & \\
\hline Age, years & 1.86 & $<0.001$ & 1.490 & 2.320 \\
\hline $12-13$ vs $14-15$ & 0.76 & 0.02 & 0.599 & 0.650 \\
\hline $12-13$ vs $16-17$ & 0.79 & 0.06 & & \\
\hline Adult education & 0.76 & $<0.001$ & 0.669 & 0.855 \\
\hline$<$ High school vs high school/diploma & 0.87 & 0.37 & & \\
\hline$<$ High school vs some college & 0.63 & 0.02 & 0.423 & 0.937 \\
\hline$<$ High school vs college or higher & 0.42 & $<0.001$ & 0.309 & 0.548 \\
\hline$\%$ of federal poverty level & 0.80 & $<0.001$ & 0.716 & 0.889 \\
\hline $0-99$ vs $\geq 300$ & 1.32 & 0.11 & & \\
\hline $100-199$ vs $\geq 300$ & 1.69 & $<0.001$ & 1.300 & 2.200 \\
\hline $200-299$ vs $\geq 300$ & 1.89 & 0.001 & 1.300 & 2.760 \\
\hline
\end{tabular}

$* p<0.05$

panics, as well as African American and Asian American adolescents (Eaton et al. 2006; Gordon-Larsen et al. 1999). Despite this obvious ethnic disparity, few published reports describe studies specific to physical activity in Hispanic American and Asian American adolescents (ADA 2006).

The different factors that contribute to overweight status may be due to our over sampling of the Asian American subgroups and to the diverse population of California. This study is unique in looking at sub-groups in California adolescents, which has not been done in the research to date. These findings are significant because when developing interventions for adolescents, it is important to consider the possible variation in factors that contribute to overweight status based on their gender and ethnicity.

Strengths, limitations, and recommendations

The strength of this study is the large sample size, which allowed analysis of multiple variables that could contribute to overweight in the ethnically diverse adolescents in California. Limitations of this study included self-reporting and the potential recall bias. Further research that uses objective measures of physical activity and body composition must be done in the area of gender and ethnic disparities as they relate to overweight. A better understanding of the differences that exist in the factors contributing to overweight in adolescents is necessary before we can develop interventions to address this growing public health issue.

\section{Conclusions}

The main finding in this study was that gender and ethnic variations are apparent in factors that contribute to overweight in California adolescents. A higher percentage of Latino adolescents, especially boys, are overweight, whereas a large proportion of Asian adolescents participate in excessive sedentary activity and limited physical activity.

A better understanding of the differences among these various sub-groups is important for the development of interventions. Disease states seen in children related to overweight status include precursors of type 2 diabetes, cardiovascular disease, sleep disorders, and metabolic syndrome (Daniels 2006). Current research continues to explore the strong correlation between genetic predisposition and variations in metabolic function in certain ethnic groups. According to Wang and Beydoun (2007), diabetes will develop at some point in half of the Latinos born today as a result of obesity.

Multiple and varying factors contribute to overweight in the ethnically diverse adolescent population of California. Published reports suggest that interventions to help address the issue of overweight in adolescents may need to be 
specific to gender and or ethnicity, and our findings support that approach. To reduce the current obesity epidemic among adolescents, clinicians and researchers must develop culturally sensitive and gender-specific interventions that address the unique needs of an ethnically diverse adolescent population. Future research should include African American adolescents since they are also at high risk for obesity, similar to Latino and Asian youth. More research also must be done to determine the factors related to increased BMI over time in adolescents and their relationship with gender and ethnicity.

Conflict of interest The authors have no relevant associations that might pose a conflict of interest.

Open Access This article is distributed under the terms of the Creative Commons Attribution Noncommercial License which permits any noncommercial use, distribution, and reproduction in any medium, provided the original author(s) and source are credited.

\section{References}

American Dietetic Association (ADA) (2006) Individual-, family-, school-, and community-based interventions for pediatric overweight. J Am Diet Assoc 106:925-945

AskCHIS (2009) Available via: http://www.chis.ucla.edu/main/ default.asp. Accessed 12 Mar 2009

Atabek ME, Pirgon O, Kurtoglu S (2006) Prevalence of metabolic syndrome in obese Turkish children and adolescents. Diabetes Res Clin Pract 72(3):315-321

Berkey CS, Rockett HRH, Gillman MW et al (2003) Longitudinal study of skipping breakfast and weight change in adolescents. Int J Obes 27:1258-1266

Boutelle K, Neumark-Sztainer D, Story M, Resnick M (2002) Weight control behaviors among obese, overweight, and nonoverweight adolescents. J Pediatr Psychol 27(6):531-540

Brown ER, Holtby S, Zahnd E, Abbott GB (2005) Community-based participatory research in the California health interview survey. Prev Chronic Dis 2(4):1-8

California Health Interview Survey (CHIS) (2007a) CHIS 2005 methodology series: report 1-sample design. UCLA Center for Health Policy Research, Los Angeles

California Health Interview Survey (CHIS) (2007b) CHIS 2005 methodology series: report 4-response rates. UCLA Center for Health Policy Research, Los Angeles

California Health Interview Survey (CHIS) (2007c) CHIS 2005 methodology series: report 2-data collection methods. UCLA Center for Health Policy Research, Los Angeles

Centers for Disease Control and Prevention (CDC)(2009) National Center for Health Statistics: 2000 CDC Growth Charts-United States. Available via: http://www.cdc.gov/growthcharts/. Accessed 16 Mar 2009

Centers for Disease Control and Prevention (CDC) (2009) Overweight and obesity. Available via: http://www.cdc.gov/nccdphp/dnpa/ obesity/index.htm. Accessed 13 Mar 2009

Centers for Disease Control and Prevention (CDC) (2009) VERB: Going behind the scenes. Available via: http://www.cdc.gov/ youthcampaign/pressroom/articles/behind-the-screen.htm. Accessed 16 Mar 2009
Chen JL (2009) Household income, maternal acculturation, maternal education level and health behaviors of ChineseAmerican children and mothers. J Immigr Minor Health (in press). doi:10.1007/s100903-008-9124-8

Daniels SR (2006) The consequences of childhood overweight and obesity. Future Child 16(1):47-67

Davison KK, Birch LL (2001) Childhood overweight: a contextual model and recommendations for future research. Obes Rev 2:159-171

Drewnowski A, Damon N (2005) The economics of obesity: dietary energy density and energy cost. Am J Clin Nutr 82(1 suppl): S265-S273

Eaton DK, Kann L, Kinchen S et al (2006) Youth risk behavior surveillance- United States, 2005. MMWR Surveill Summ 55 (5):1-108

Freedman DS, Wang J, Thornton JC et al (2008) Racial/ethnic differences in body fatness among children and adolescents. Obesity 16(5):1105-1111

Fulkerson JA, Neumark-Sztainer D, Hannan PJ et al (2007) Family meal frequency and weight status among adolescents: crosssectional and 5-year longitudinal associations. Obesity 16:25292534

Gable S, Chang Y, Krull JL (2007) Television watching and frequency of family meals are predictive of overweight onset and persistence in a national sample of school-aged children. J Am Diet Assoc 107(1):53-61

Gordon-Larsen P, McMurray RG, Popkin BM (1999) Adolescent physical activity and inactivity vary by ethnicity: the national longitudinal study of adolescent health. J Pediatr 135:301-306

Gordon-Larsen P, Adair LS, Popkin BM (2003) The relationship of ethnicity, socioeconomic factors, and overweight in U.S. adolescents. Obes Res 11(1):121-129

Holtby S, Zahnd E, Chia YJ et al (2008) Health of California's adults, adolescents and children: findings from the CHIS 2005 and CHIS 2003. UCLA Center for Health Policy Research, Los Angeles

Laustsen G (2006) Environment, ecosystems and ecological behavior: a dialogue toward developing nursing ecological theory. Adv Nurs Sci 29(1):43-54

Lopez-Zetina J, Lee H, Friis R (2006) The link between obesity and the built environment: evidence from an ecological analysis of obesity and vehicle miles of travel in California. Health Place 12 (4):656-664

Lustig RH (2006) Childhood obesity: behavioral aberration or biochemical drive? Reinterpreting the first law of thermodynamics. Endocrinol Metab 2(8):447-458

Must A, Bandini LG, Tybor DJ et al (2007) Activity, inactivity, and screen time in relation to weight and fatness over adolescence in girls. Obesity 23(suppl. 2):s2-s11

Nader PR, Bradley RH, Houts RM et al (2008) Moderate-to-vigorous physical activity from age 9 to 15 years. JAMA 300(3):295-305

Neumark-Sztainer DR, Hannan PJ, Story M et al (2003) Family meal patterns: association with sociodemographic characteristics and improved dietary intake among adolescents. J Am Dietetic Assoc 103:317-322

Neumark-Sztainer DR, Wall MM, Haines JI et al (2007) Shared risk and protective factors for overweight and disordered eating in adolescents. Am J Prevent Med 33(5):359-369

Ogden CL, Carroll MD, Curtin LR et al (2006) Prevalence of overweight and obesity in the United States, 1999-2004. JAMA 295(13):1549-1555

Ogden CL, Carroll MD, Flegal KM (2008) High body mass index for age among U.S. children and adolescents, 2003-2006. JAMA 299(20):2401-2405

Parizkova J (2008) Impact of education on food behavior, body composition and physical fitness in children. Br J Nutr 99(suppl 1):s26-s32 
Pearson N, Biddle SJH, Gorely T (2009) Family correlates of breakfast consumption among children and adolescents: a systematic review. Appetite 52:1-7

Perry AC, Rosenblatt EB, Wang X (2004) Physical, behavioral, and body image characteristics in a tri-racial group of adolescent girls. Obes Res 12(10):1670-1679

Ponce NA, Lavarreda SA, Yen W et al (2004) The California health interview survey 2001: translation of a major survey for California's multiethnic population. Pub Health Rep 119:388-395

Popkin BM, Udry JR (1998) Adolescent obesity increases significantly in second and third generation U.S. immigrants: the national longitudinal study of adolescent health. J Nutr 128(4):701-706

Rowland, TW (1999) Adolescence: A "risk factor" for physical inactivity. President's council on physical fitness and sports research digest. Available via http://purl.access.gpo.gov/GPO/ LPS21085 Accessed 10 Aug 2007

Sallis JF, Owen N (1997) Ecological models. In: Glanz K, Lewis FM, Rimer BK (eds) Health behavior and health education: theory, research and practice, 2nd edn. Jossey-Bass, San Francisco, pp $403-424$
Scharoun-Lee M, Adair LS, Kaufman JS et al (2009) Obesity, race/ ethnicity, and the multiple dimensions of socioeconomic status during the transition to adulthood: a factor analysis approach. Soc Sci Med. doi:10.1016/j.socscimed.2008.12.009

Sinha A, King S (2008) A review of adolescent obesity: prevalence, etiology, and treatment. Obesity Surg. 19:113-120

Timlin MT, Pereira MA, Story M et al (2008) Breakfast eating and weight change in a 5-year prospective analysis of adolescents: project EAT (Eating Among Teens). Pediatrics 121(3):e638-e645

Wang Y, Beydoun MA (2007) The obesity epidemic in the United States - gender, age, socioeconomic, racial ethnic, and geographic characteristics: a systematic review and meta-analysis. Epidemiol Rev 29:6-28

Woodruff SJ, Hanning RM (2009) Associations between family dinner frequency and specific food behaviors among grade six, seven, and eight students from Ontario and Nova Scotia. doi:10.1016/ jadohealth.2008.10.141

Zhang Q, Wang Y (2004) Trends in the association between obesity and socioeconomic status in U.S. adults: 1971 to 2000. Obes Res 12(10):1622-1632 